\title{
How Reliably Does a Neuron in the Visual Motion Pathway of the Fly Encode Behaviourally Relevant Information?
}

\author{
Anne-Kathrin Warzecha and Martin Egelhaaf \\ Lehrstuhl für Neurobiologie, Fakultät für Biologie, Universität Bielefeld, Postfach 1001 31, D-33501 Bielefeld, Germany \\ Most of the experiments were performed at the Centre for Visual Science (Australian National University, Canberra, Australia)
}

Keywords: Lucifia cuprina, neural code, reliability, visual motion

\begin{abstract}
How reliably neurons convey information depends on the extent to which their activity is affected by stochastic processes which are omnipresent in the nervous system. The functional consequences of neuronal noise can only be assessed if the latter is related to the response components that are induced in a normal behavioural situation. In the present study the reliability of neural coding was investigated for an identified neuron in the pathway processing visual motion information of the fly (Lucilia cuprina). The stimuli used to investigate the neuronal performance were not exclusively defined by the experimenter. Instead, they were generated by the fly itself, i.e. by its own actions and reactions in a behavioural closed-loop experiment, and subsequently replayed to the animal while the activity of an identified motion-sensitive neuron was recorded. Although the time course of the neuronal responses is time-locked to the stimulus, individual response traces differ slightly from each other due to stochastic fluctuations in the timing and number of action potentials. Individual responses thus consist of a stimulus-induced and a stochastic response component. The stimulus-induced response component can be recovered most reliably from noisy neuronal signals if these are smoothed by intermediate-sized time windows (40-100 ms). At this time scale the best compromise is achieved between smoothing out the noise and maintaining the temporal resolution of the stimulus-induced response component. Consequently, in the visual motion pathway of the fly, behaviourally relevant motion stimuli can be resolved best at a time scale where the timing of individual spikes does not matter.
\end{abstract}

\section{Introduction}

Even when navigating through hectic everyday traffic we usually take it for granted that we will be able to respond appropriately in most situations and thus arrive safely at our destination. In many cases, this ability requires fast behavioural decisions which, notwithstanding, have to be sufficiently reliable. Such decisions are inevitably preceded within the organism by a series of processing steps. First, the outside world has to be perceived by the sensory system. Then the relevant features of the stimuli have to be extracted in the nervous system from the activity profile of the sensory neurons before the appropriate motor programs can be initiated to execute the final behavioural reactions. Apart from the fact that all these processing steps may take a considerable amount of time, they do not follow completely deterministic rules, and thus may lead to a somewhat unpredictable outcome of the whole sequence of information processing. Consequently, behavioural responses have only limited reliability. This indeterminacy may be a consequence of noisy sensory input (e.g. photon noise: Bouman et al., 1985), synaptic noise (e.g. Allen and Stevens, 1994), or the stochastic opening and closing of ionic channels underlying neuronal activity (Hille, 1992). Thus, when a given stimulus is presented repeatedly to a neuron, its responses are by no means identical but may vary quite a lot. It is not uncommon that the variance of these stochastic neuronal response fluctuations is almost as large as the average response amplitude (e.g. Tolhurst $e t$ al., 1983; Vogels et al., 1989; Miller et al., 1991; Britten et al., 1993).

How does the nervous system cope with the detrimental consequences of its various noise sources? Experimenters usually try to eliminate noise from the neuronal response traces in order to increase the reliability of their inferences about stimulus-response relationships by averaging the individual responses to many stimulus presentations. However, in real life an animal can hardly ever employ this strategy. As humans do in daily traffic, an animal often has only one chance to react correctly. Hence, in order to understand how the nervous system is able to mediate behavioural responses in real time, it is essential to take into account the functional consequences of the limited reliability of neural signals.

Of course, the absolute noise level on its own does not tell much about how reliably information is encoded by a neuron. Rather, how well a neuron can convey information depends in an intricate way

Correspondence to: A. $\mathrm{K}$. Warzecha, as above

Received 8 August 1996, revised 16 December 1996, accepted 6 February 1997 
on the relationship between the amplitude of the noise and that of the stimulus-induced response component ('SIRC'). In a normal behavioural situation the animal's own actions and reactions have immediate consequences on its sensory input. Therefore, the SIRC is not simply the consequence of an external source but is determined, at least to a large extent, by the animal itself. Hence, in order to assess the functional significance of neuronal noise, the noise has to be related to the stimulus-induced responses as are elicited by behaviourally relevant stimuli, i.e. stimuli that are similar to those experienced by an animal in a normal behavioural situation.

The relationship between stochastic and stimulus-induced response components may greatly depend upon the temporal resolution with which the neuronal responses are taken into account. For instance, if responses are smoothed by temporal integration within a time window of a given size, both the noise as well as the SIRC may decrease, though to a different extent depending on the size of the integration window, On which time scale neuronal activity in a particular system conveys most information cannot be decided solely by inspecting neuronal responses, but can only be inferred if the constraints are known that are imposed by the computational task of the system.

In the present account the precision of neural coding is analysed in the context of visual orientation behaviour of the fly. Flies are able to exhibit perfectly visually controlled flight manoeuvres which can be analysed both under free-flight conditions (Land and Collett, 1974; Wagner, 1986; Kimmerle et al., 1996) as well as with a flight simulator under well defined laboratory conditions (Reichardt and Poggio, 1976; Heisenberg and Wolf, 1984; Egelhaaf et al., 1988; Egelhaaf and Borst, 1993a). In order to be able to perform these flight manoeuvres, the animals have to stabilize their course against disturbances, such as a gust of wind. Deviations from a straight flight course lead to a characteristic optic flow on the eyes which contains a strong rotational component. This rotational component is evaluated in the fly's nervous system and transformed into compensatory steering movements which stabilize the flight course.

Visual interneurons in the third visual neuropil which are specifically tuned to the optic flow generated on the eye during visual orientation behaviour are readily accessible to experimental analysis (for review see: Hausen, 1981; Egelhaaf et al. 1988; Hausen and Egelhaaf, 1989; Egelhaaf and Borst, 1993a). In particular, one of these neurons, the so-called H1-cell, can be recorded from for extended periods of time. The H1-cell responds best to coherent motion in large parts of the visual field and plays a role in tuning other visual interneurons to particular types of optic flow (Hausen, 1981; Hausen and Egelhaaf, 1989). Since the activity of this neuron can be manipulated in a well defined way by appropriate visual stimulation, the reliability of neuronal responses contingent upon a given stimulus can be assessed. Hence, as has been shown in previous studies (Gestri et al., 1980; Ruyter van Steveninck and Bialek, 1988, 1995; Bialek et al. 1991), the H1-cell lends itself well to an experimental analysis of how precisely a stimulus can be encoded by a neuron. In contrast to these studies, we analysed in the present account the consequences of neuronal noise for the representation of behaviourally relevant stimuli by the H1-cell. By 'behaviourally relevant', we mean that the stimuli used in the electrophysiological experiments were generated in previous behavioural experiments with a flight simulator by the fly's own actions and reactions while it tried to stabilize its flight course in a similar way as in natural flight. With this approach we try to answer two specific questions. (i) How precisely do neuronal responses represent behavjourally relevant information? (ii) What is the time scale on which the stimulus-induced response component can be recovered most reliably from the noisy neuronal signals?

\section{Materials and methods}

\section{Preparation and electrophysiology}

For electrophysiological experiments female blowflies (Lucilia cuprina) were obtained from stocks raised by CSIRO, Canberra (Australia). The animals were anaesthetized briefly with $\mathrm{CO}_{2}$ and mounted, ventral side up, with wax on a small piece of glass. The legs were amputated and the head was tilted about $30^{\circ}$ ventrally and waxed to the thorax. The head capsule was opened from behind and the trachea and air sacs covering the brain were removed to gain access to the posterior part of the third visual neuropil. To avoid desiccation of the brain, the head capsule was supplied with Ringer solution (for composition, see Hausen, 1982). The experiments were performed at room temperature $\left(19-22^{\circ} \mathrm{C}\right)$.

Tungsten electrodes were used for extracellular recording of $\mathrm{H} 1$ cells. Their tips were sharpened electrolytically and insulated with varnish. They had resistances between 2 and $8 \mathrm{M} \Omega$. Recorded signals were amplified by standard electrophysiological equipment. Spikes were transformed into pulses of fixed height and duration. Signals were fed to a 486-PC through an analogue-to-digital converter of an I/O-card (2801A, Data Translation, Marlboro, MA). The temporal resolution amounted to $5 \mathrm{~ms}$. Controls were performed to ensure that every spike was counted.

\section{Visual stimulation in the experiments using behaviourally relevant stimuli}

Two CRT screens (Tektronix 608, Wilsonville, OR) were used for visual stimulation. The stimulus pattern was generated at a frame rate of $183 \mathrm{~Hz}$ by an image synthesizer (Picasso, Innisfree, Cambridge, MA). Monitors were placed symmetrically at an angle of $45^{\circ}$ with respect to the long axis of the fly and perpendicular to the horizontal plane of the animal. The centres of the screens were placed at angular horizontal positions of $+55^{\circ}$ or $-55^{\circ}$ (right and left screen respectively) and at an angular vertical position of $0^{\circ}$. The horizontal and vertical extent of the screens amounted to $70^{\circ}$ and $55^{\circ}$ respectively. The stimulus pattern consisted of a vertical black and green square-wave grating with five periods per screen. The mean luminance was $44 \mathrm{~cd} /$ $\mathrm{m}^{2}$, the contrast 0.92 . The time dependent displacements of the stimulus gratings that were used as motion stimuli in the electrophysiological experiments were obtained in a previous behavioural analysis where tethered flies flying in a flight simulator were allowed to influence their visual input by their own actions and reactions. This was accomplished by measuring the torque responses of the tethered flying fly with a torque compensator and feeding them back to the image synthesizer, thus, allowing them to affect the displacements of the stimulus pattern in a similar way as in a natural situation. An intended turn of the fly in one direction induced a displacement of the pattern in the opposite direction with a velocity proportional to the torque signal. During part of each behavioural closed-loop experiment an external motion bias was added to the pattern displacement induced by the fly's torque signal. In an open-loop situation this bias would have displaced the stimulus pattern with a constant velocity. The motion bias was superimposed, to test how well flies can stabilize their flight path against external disturbances. The analysis of the behavioural responses and corresponding motion traces is presented elsewhere (Warzecha, 1994; Warzecha and Egelhaaf, 1996). It had been established that the dynamics of the torque meter and associated electronic system do not significantly affect the dynamics of the pattern displacement (Warzecha and Egelhaaf, 1996). The torque signals were stored in the memory of a computer for later data analysis and for replay of the same stimuli in subsequent electrophysiological experiments. In order to investigate the responses 
of the H1-neuron to these stimuli, two typical motion traces generated by different flies were selected arbitrarily and presented in subsequent experiments to three cells between 30 and 60 times. Each motion trace consisting of two sections lasted for $37.5 \mathrm{~s}$, only $29.35 \mathrm{~s}$ of which were included in the determination of the transinformation (Figs 3-5). The first part of the neuronal response within each section was excluded from the analysis to avoid transient effects. For calculating the power density spectra (Fig. 2) only the last $10.24 \mathrm{~s}$ of each section were analysed. This interval length was chosen because it corresponds to a number of data points amounting to an integral power of two which is required for the Fast-Fourier algorithm. Between consecutive presentations of the same stimulus the grating remained stationary while individual response traces were stored on the PC. Data acquisition and evaluation were carried out with a 486 PC. The programs were written in ASYST (Keithley Instruments, Taunton, MA).

\section{Results}

\section{Stimulus-induced and stochastic signal fluctuations}

In the present account it was intended to find out how well the H1cell, a spiking neuron in the fly's visual motion pathway, encodes behaviourally relevant motion stimuli and to what extent the representation of such stimuli is affected by stochastic signal fluctuations. Therefore, the H1-cell was stimulated by motion traces that were generated in a previous behavioural experiment by a tethered flying fly in a flight simulator under closed-loop conditions. This procedure is illustrated in Figure 1. At first, spontaneous turning responses of the tethered fly were recorded with a torque meter, processed in a computer and subsequently fed back to displace the stimulus grating. The pattern, thus, moved to the left when the fly tried to turn to the right and vice versa. Then a disturbance was added for a given time interval to the visual input signal generated by the fly's spontaneous actions. In an open-loop situation, this disturbance would have displaced the stimulus pattern with a constant velocity in one direction. Following this interval, the disturbance was switched off again. Although on average flies compensate an external disturbance such as the motion bias to a large extent, the individual motion traces are characterized by pronounced velocity fluctuations. These fluctuations are very large compared to the mean slip speed (for a more detailed analysis of these fluctuations and their origin see Warzecha, 1994; Warzecha and Egelhaaf, 1996). As a consequence, it is hardly possible to discern on the basis of individual motion traces whether or not these were generated while an external motion bias was added to the visual input. This suggests that the time course of the retinal image motion is mainly characterized by the fly's own actions and reactions.

Typical motion traces generated by the fly under closed-loop conditions were replayed to the animal many times while recording the activity of the H1-cell. The diagram in the left middle part of Figure 1 depicts consecutive individual responses of the neuron to subsequent presentations of such a motion trace. Although the stimulus was the same for each response trace and the response traces reveal an overall similarity, they are not identical but show some variability. Hence, neuronal responses consist of two components, one that is induced by the stimulus and free of accidental events as well as another component that is not under the control of the experimenter. The latter component comprises all true stochastic signal components but also those which are not locked to the stimulus and, nonetheless, might be due to some unknown deterministic source. Since these can hardly be disentangled, they will be collectively referred to in the following as 'stochastic response component'. By averaging many responses to an identical motion trace, the stochastic signal fluctuations are eliminated and the spike frequency histogram is obtained (Fig. 1, bottom diagram of left column). This spike frequency histogram is assumed to represent the SIRC. The average response of the directionally selective $\mathrm{H} 1$-cell is strongly modulated by the profound fluctuations in the velocity of the stimulus. As long as the pattern moves in the cell's preferred direction, the neuronal response follows, to some extent, the retinal slip speed with a phase shift (see also Bialek et al., 1991). It should be noted that the time course of the response does not only depend on the velocity but also on its higher temporal derivatives (see Egelhaaf and Reichardt, 1987; Egelhaaf and Borst, 1993b). In any case, the neuronal responses are to some degree timelocked to the stimulus. Of course, there is a time shift between stimulus and response which was determined by cross-correlating the velocity and the corresponding SIRC. The cross-correlogram has its maximum at $60 \mathrm{~ms}$ (Warzecha and Egelhaaf, 1996).

The velocity fluctuations and the fluctuations in the SIRC are reflected in the corresponding power spectra. An example is illustrated in Figure 2. In the power spectrum of the velocity trace the lowest frequencies that can be determined within the analysed time interval of $10.24 \mathrm{~s}$ predominate. The remaining power is distributed mainly among frequencies up to about $5 \mathrm{~Hz}$ with a slight increase between 1.5 and $3.5 \mathrm{~Hz}$. In the power spectrum of the corresponding cellular response there is considerably less power in the very low frequency range. Instead, frequency components around $2.5 \mathrm{~Hz}$ prevail. The power spectra of the other velocity and response traces used in the experiments of the present study exhibit similar features, in particular, with respect to the decline towards high frequencies.

\section{How reliably do individual responses represent the stimulus- induced response component?}

The SIRC is a construct of the experimenter for analytical purposes and does not contain stochastic components. It is, of course, not available to the animal. Rather, all information conveyed by a neuron has to be decoded by the postsynaptic neuronal machinery from the individual response traces in real time. The decoding mechanisms, therefore, have to cope with noise that may conceal the stimulusinduced response. For the animal this will lead to the problem of how to decide to what extent a given response represents a given stimulus. In the first step of the present analysis it will be investigated by calculating the so-called transinformation: how well the information contained in the SIRC is represented by the individual responses. Quite generally, the transinformation is a measure of how well a given signal can be predicted on the basis of another signal.

According to information theory (Shannon and Weaver, 1949), the information content of a given signal amplitude $s_{\mathrm{i}}$ is related to the dyadic logarithm of the reciprocal value of its probability of occurrence $\mathrm{p}\left(s_{\mathrm{i}}\right)$. The average amount of information conveyed by all the different signal levels ('information capacity', $I$ ) is then given by the sum of the information conveyed by each signal level times that signal level's probability of occurrence

$$
\left.\mathrm{I}=\Sigma p\left(s_{\mathrm{i}}\right) \cdot \log _{2}\left[1 / p\left(s_{\mathrm{i}}\right)\right]=-\Sigma p\left(s_{\mathrm{i}}\right)\right] \cdot \log _{2}\left[p\left(s_{\mathrm{i}}\right)\right]
$$

As mentioned above, the transinformation is taken in the first step of our analysis as a measure of the information that is actually transmitted by individual response traces about the SIRC. For instance, if the individual response traces are affected by only small stochastic fluctuations, the SIRC can be predicted on their basis with a high degree of reliability. Conversely, if the individual responses are not much correlated with the corresponding stimulus-induced signal, the latter can hardly be predicted on the basis of an individual response 


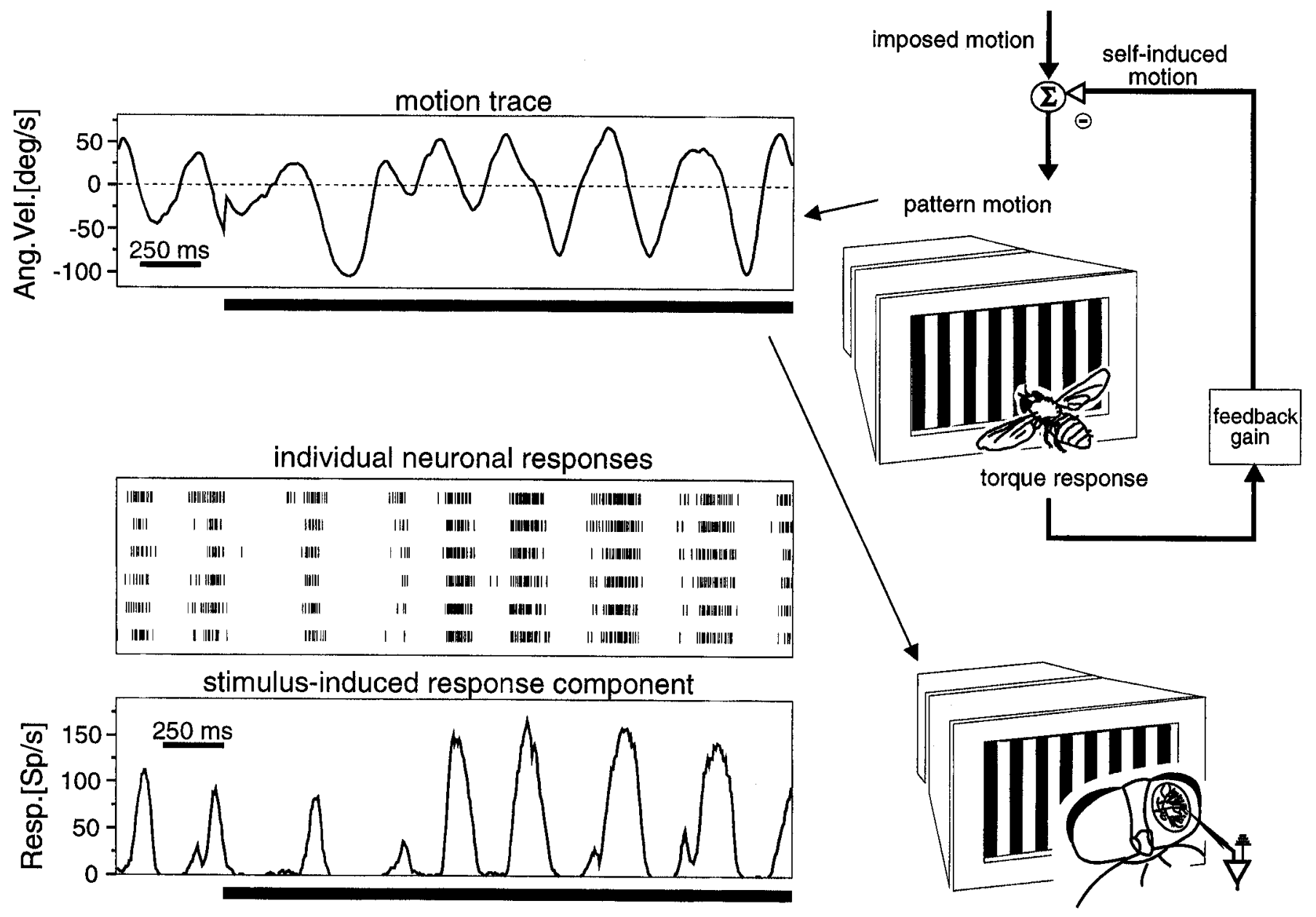

FIG. 1. Generation of behaviourally relevant motion traces and corresponding neuronal responses. Upper right: Behavioural closed-loop situation. The fly is tethered to a torque compensator (not shown) which makes it possible to measure the fly's instantaneous torque. In contrast to free flight, the torque signal generated by the fly does not lead to turns of the animal that normally would induce retinal image displacements. Rather, the visual consequences of self-motion are simulated by feeding the torque signals into a computer and transforming them into image displacements on two CRT screens (only one is shown). The image displacements are assumed to be proportional to the torque. In addition to the self-induced image displacements, an additional motion bias can be added to the fly's visual input. Upper left: time course of a velocity trace generated in the behavioural closed-loop situation. For the duration indicated by the black bar underneath the velocity trace an external motion bias with a constant velocity of $44 \%$ was added to the pattern displacements induced by the fly's own torque. Lower right: the motion was replayed in a subsequent electrophysiological experiment to a fly while recording the activity of an H1-cell in the right half of the brain. Middle left: six consecutive unaveraged neuronal responses to identical stimulation with the motion trace shown above plotted underneath each other. The occurrence of a spike is indicated by a small vertical line. Lower left: stimulus induced response component (SIRC) as obtained by averaging 40 individual responses to identical stimulation. The black bar underneath the response trace indicates the time when in the behavioural closed-loop experiment the external motion bias was added to the fly's own torque signal.

trace. In the latter case the transinformation will therefore be smaller than in the first example. Hence, the transinformation is related to the conditional probability of a particular level of the SIRC given a particular amplitude of an individual response $p\left(s_{\mathrm{i}} \mid r_{\mathrm{j}}\right)$. The transinformation $(T)$ is then given by the formula (Shannon and Weaver, 1949; Eckhorn and Pöpel, 1974)

$$
T=\Sigma \Sigma p\left(s_{\mathrm{i}}, r_{\mathrm{j}}\right) \cdot \log _{2}\left[p\left(s_{\mathrm{i}} \mid r_{\mathrm{j}}\right) / p\left(s_{\mathrm{i}}\right)\right]
$$

with $p\left(s_{\mathrm{i}}, r_{\mathrm{j}}\right)$ representing the joint probability of a given level $s_{\mathrm{i}}$ of the SIRC and a particular amplitude $r_{j}$ of the individual responses. If the SIRC can be predicted with absolute certainty on the basis of an individual response, $p\left(s_{\mathrm{i}} \mid r_{\mathrm{j}}\right)=1$ and Equation 2 reduces to the information capacity given in Equation 1, implying that the complete information of the stimulus-induced response is conveyed by each individual response.

To derive the probability distributions that are required to calculate the transinformation conveyed by individual response traces about the SIRC, the following steps were employed. (i) Individual responses were determined by counting spikes in consecutive $5 \mathrm{~ms}$ time windows. (ii) The sample average of many individual responses served as an estimate of the SIRC. (iii) The transinformation can be expected to depend on the temporal resolution chosen for the analysis. In order to investigate at what time scale most information can be obtained from individual responses about the SIRC, the analysis was, therefore, performed by integrating the individual responses within time windows between 5 and $640 \mathrm{~ms}$. These integration windows were shifted in time steps of $5 \mathrm{~ms}$ along the response traces. (iv) To obtain the probability distributions required for calculating Equation 2 , both the stimulus-induced response with a time resolution of $5 \mathrm{~ms}$ as well as the individual responses evaluated with differently sized time windows had to be subdivided into discrete response classes. These classes were chosen to have a width of 10 spikes/s. Since 

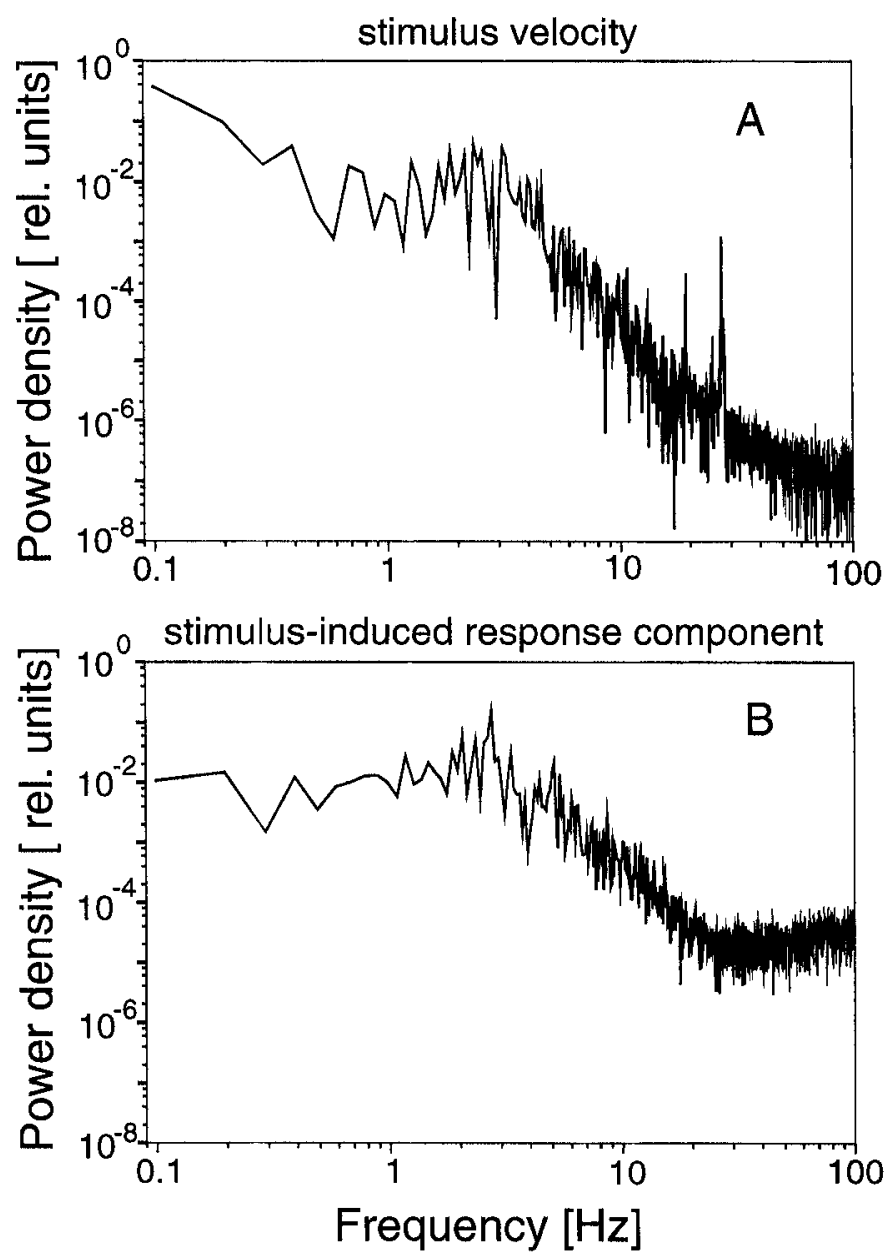

FIG. 2. Power density spectrum of (A) the velocity of a motion trace as was generated by a fly in a behavioural closed loop experiment and (B) of the stimulus-induced component of the corresponding neuronal response. Power spectra were calculated by a Fast-Fourier algorithm and normalized to a total power of 1. The data shown in (B) represent the mean of the power spectra obtained from two flies. The corresponding SIRCs are based on 30 and 40 individual response traces respectively. Signals within two time intervals of $10.24 \mathrm{~s}$ each were taken into account. This interval was chosen because it corresponds to a number of data points amounting to an integral power of two, which is required for the Fast-Fourier algorithm. Whereas, the power spectrum of the velocity trace assumes its highest values at very low frequencies, the SIRC has its most prominent frequency components between 2 and $3 \mathrm{~Hz}$. High frequency components have only little power in both the velocity trace and the SIRC.

this choice of the size of the subdivisions is rather arbitrary, the transinformation was also calculated for activity classes of 5 and 20 spikes/s as a control. (v) For each H1-cell that was analysed, the whole ensemble of individual response traces obtained by repeatedly presenting the same motion trace was subdivided into two sets. Every second sweep was selected for one set while the remaining sweeps constituted the other set. For each set a separate SIRC was determined and used for calculating the transinformation on the basis of the individual response traces that constitute the other set. In this way, two transinformation values were obtained for each H1-cell and each time window. The mean of these corresponding pairs of values will be referred to below. This procedure of splitting up the data into two sets was necessary because otherwise each individual response would have been correlated with the SIRC simply because it is part of the latter.

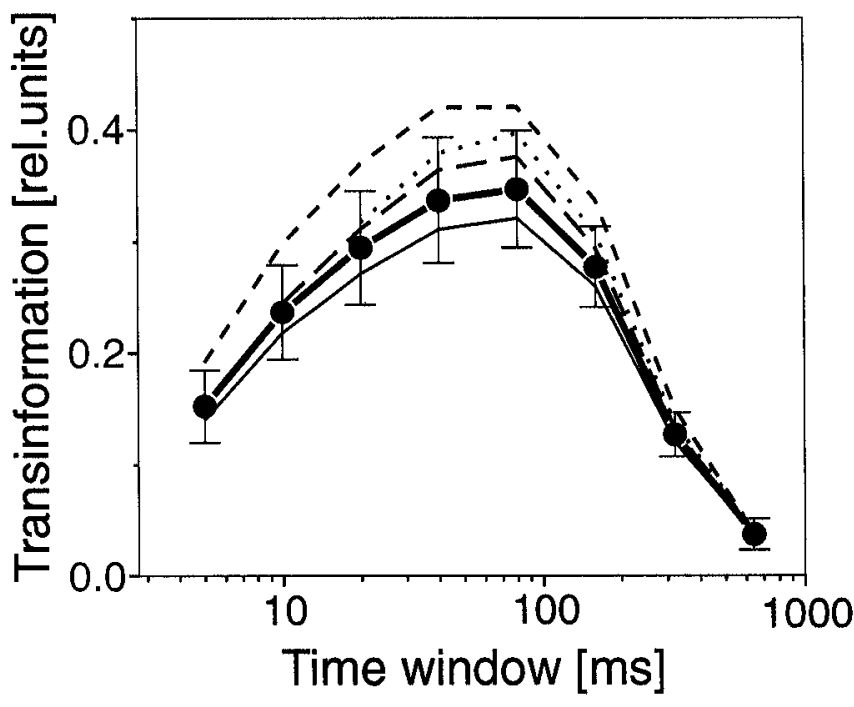

Fig. 3. Transinformation conveyed by individual neuronal responses about the SIRC as a function of the time window used for integrating the individual responses. Different line styles illustrate the result of varying the exact procedure to calculate the transinformation. The thick solid line connecting the filled circles has been obtained by determining the SIRC on the basis of spike counts within consecutive $5 \mathrm{~ms}$ windows. Spike counts for individual responses as well as for the SIRC were subdivided into activity classes of 10 spikes/s to obtain the probability distributions for calculating the transinformation. To test how the shape of the curve depends on the temporal resolution of the SIRC or the size of the activity classes, these parameters were varied (lower dashed line: temporal resolution of SIRC $-10 \mathrm{~ms}$, size of activity classes - 10 spikes/s; dotted line: temporal resolution of SIRC - 20 $\mathrm{ms}$, size of activity classes - $10 \mathrm{spikes} / \mathrm{s}$; solid thin line: temporal resolution of SIRC $-5 \mathrm{~ms}$, size of activity classes -5 spikes/s; upper dashed line: temporal resolution of SIRC $-5 \mathrm{~ms}$, size of activity classes -20 spikes/s). Note that the leftmost data point of each line coincides with the size of the time window used for calculating the corresponding SIRC (e.g, $584 \mathrm{bit} / \mathrm{s}$ for the thick solid curve). Each of the curves was normalized to the information capacity of the corresponding SIRC. Lines connect mean values for the transinformation obtained from three flies and a total of 130 individual response traces of $29.35 \mathrm{~s}$ duration each; error bars denote corresponding SEMs. For details of the analysis see text.

It should be noted that the transinformation is a highly non-linear measure of how well a given signal can be predicted on the basis of another signal. This non-linearity is particularly obvious for a twoalternative-forced-choice situation. If, for instance, an observer decides correctly in $90 \%$ of all cases, the transinformation he/she conveys about the signal decreases to about $50 \%$ as compared to the transinformation obtained with $100 \%$ correct performance. This relation should be kept in mind when the following data are interpreted in quantitative terms.

In Figure 3 the transinformation conveyed by individual response traces is plotted as a function of the time window with which they were evaluated. Since the absolute figures of the transinformation do not immediately make it possible to draw conclusions about how well individual responses represent the SIRC, the transinformation was normalized to the information capacity of the SIRC as determined according to Equation 1. Moreover, since the transinformation depends on the temporal resolution with which the SIRC is determined as well as on the graduation of the probability distributions, these parameters were varied (different line styles in Fig. 3). Independent of the exact choice of parameters the transinformation increases at first with increasing time window, reaches a maximum for a window size between $40 \mathrm{~ms}$ and $80 \mathrm{~ms}$ and then decreases again. It is obvious from Figure 3 that even for the best time window only about 35- 
$40 \%$ of the information contained in the SIRC is transmitted by individual responses. The thick solid line connecting the circles illustrates the transinformation obtained for activity classes of 10 spikes/s and a temporal resolution of the SIRC of $5 \mathrm{~ms}$. When, as a control, the probability distributions for calculating the transinformation were determined on the basis of responses that were subdivided into larger or smaller activity classes (five spikes/s, 20 spikes/s), or when the elementary time window with which the SIRC was obtained was enlarged to either $10 \mathrm{~ms}$ or $20 \mathrm{~ms}$, the shape of the curve shown in Figure 3 does not change qualitatively. Most importantly, its maximum stays between $40 \mathrm{~ms}$ and $80 \mathrm{~ms}$. Only the absolute figures of the transinformation and the information capacity to which the former has been normalized vary slightly due to the change in the probability distributions and the number of different response levels obtained with the different procedures. Consequently, the dependence of the relative transinformation on the size of the time window within which the neuronal activity was integrated is robust against the exact procedure used for its evaluation. Hence it can be concluded that information about the SIRC can be recovered best from individual real-time responses, if these are smoothed by time windows of considerable length.

This finding may result from two antagonistic effects. On the one hand, it is expected that the stochastic response components are smoothed out by increasing the size of the time window within which individual responses are integrated. Thus, temporally filtering individual response traces with increasingly larger time windows should result in an increase of the transinformation due to a reduction of neuronal noise. On the other hand, when the time windows used for assessing individual responses are enlarged further and further, the SIRC will eventually be smoothed out. Thus, one would expect that smoothing individual responses with increasingly larger time windows will reduce the information that can be conveyed by individual responses about the SIRC. We again used the transinformation as a measure to quantify these two opposing effects.

The consequences of smoothing the SIRC were assessed by calculating the information that is conveyed by temporally filtered versions of the SIRC about the unsmoothed version. Therefore, $p\left(r_{\mathrm{j}}\right)$ in Equation 2 now represents the probability of a given response level of the SIRC as smoothed by integrating the responses within differently sized time windows that were slid over the time-dependent responses in time steps of $5 \mathrm{~ms} . p\left(s_{\mathrm{i}}\right)$ still has the same meaning as defined above. As shown previously, the transinformation determined in this way was normalized to the information capacity of the unsmoothed SIRC. As expected, the information conveyed by filtered versions of the SIRC about the original, non-filtered one, decreases monotonically with increasing time window (Fig. 4).

In order to quantify the consequences of smoothing out the stochastic response component, it was determined how well individual responses filtered with time windows of variable size represent the equally filtered SIRC. Therefore, $p\left(s_{\mathbf{i}}\right)$ in Equation 2 now represents the probability of a given response level of the SIRC after smoothing it in the same way as the individual responses. The transinformation determined for cach time window was normalized to the information capacity of the respectively filtered SIRC calculated according to Equation 1, i.e. each data point in Figure 5 was normalized to a different figure. At a fine time scale the information capacity is reduced to $15 \%$ due to noise. With increasing time window the relative transinformation increases up to $50 \%$ for a $640 \mathrm{~ms}$ window (Fig. 5). Obviously, the stochastic response component gets smaller with increasing size of the time window with which it is assessed.

As a consequence of the antagonistic effects of smoothing out the

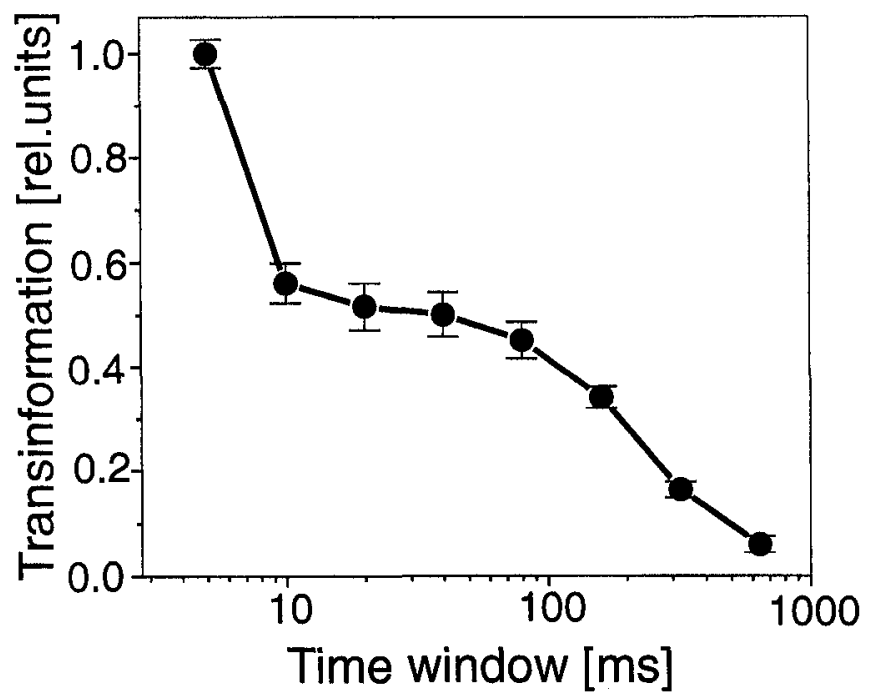

Fig. 4. Transinformation conveyed by the SIRC as smoothed by a range of time windows about the unsmoothed version, i.e. the SIRC obtained from spike counts within consecutive $5 \mathrm{~ms}$ time windows (size of activity classes: $10 \mathrm{spikes} / \mathrm{s}$ ). The transinformation was normalized to the information content of the unsmoothed stimulus-induced response. For details of the analysis see text. The same data set was used as in Figure 3. Error bars denote SEMs.

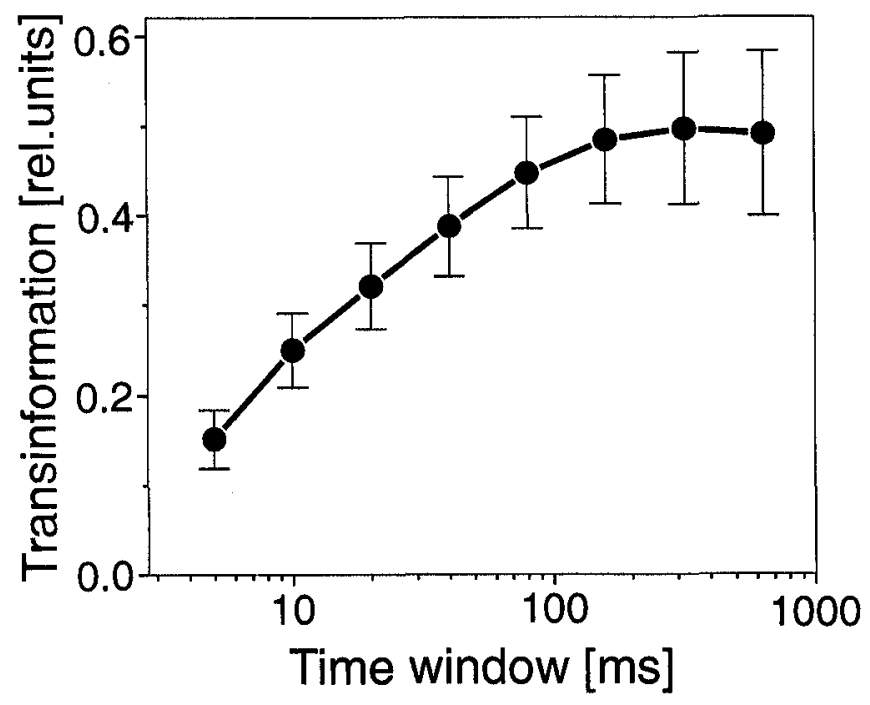

FIG. 5. Transinformation conveyed by individual responses smoothed by a range of time windows about the correspondingly smoothed SIRC (size of activity classes: 10 spikes/s). Each transinformation was normalized to the information content of the correspondingly smoothed stimulus-induced response. For details of the analysis see text. The same data set was used as in Figures 3 and 4. Error bars denote SEMs.

noise and the SIRC, most information about behaviourally relevant motion stimuli is conveyed at a time scale of about $40-100 \mathrm{~ms}$.

\section{Discussion}

Variability of neural responses appears to be abundant at all levels of nervous systems (see Introduction). Given this variability it is far from being clear what strategies are employed by nervous systems to ensure that animals can eventually perform their behavioural acts or perceptual tasks with sufficient reliability to survive in an often 
hostile environment. Put in this way, it is evident that a final assessment of the reliability of neural coding is only possible if the neuronal responses are not considered in isolation but are related to behavioural or perceptual abilities. During the last years the reliability of neural coding is being addressed in a continually increasing number of studies which scrutinize the activity patterns of neurons with a host of sophisticated techniques. In most cases, however, the results cannot directly be related to the behavioural or perceptual performance of the animal. An exception is the work on the reliability of neural coding in the motion pathway of the macaque monkey. Here the performance of neurons in detecting motion stimuli has been directly related to the concurrently determined performance of the animal in perceiving these motion stimuli (e.g. Shadlen et al., 1996). In these experiments the animal was required to inspect a visual stimulus for $2 \mathrm{~s}$ before it was allowed to make its decision. During this time interval spikes of a neuron in the motion pathway were counted. Hence, the monkey's response did not affect the visual stimulus, which was exclusively controlled by the experimenter. Though this technique has been very successful, it does not address an important aspect of the situation an animal encounters in real life. Here, quite generally, the visual input of an animal is affected by its own actions and reactions. In particular, the dynamic properties of natural stimuli as are generated on the eyes under such closed-loop conditions may differ considerably from those stimuli that are usually designed by experimenters.

For this reason, a novel approach to analysing the reliability of neural coding has been employed in the present study. Rather than activating neurons with stimuli that have been designed exclusively for analytical purposes, the neuron which has been analysed here was confronted with stimuli that were essentially generated by the behaving animal itself, i.e. by its own actions and reactions. We performed our analysis on the visuil system of the fly, where we have access to both the behavioural and the neuronal level (for review see Egelhaaf and Borst, 1993a). Here we analysed with a flight simulator the performance of the fly in optomotor course stabilization under closedloop conditions where the visual input is affected by the fly's responses in a similar way as in normal flight. Motion traces that were generated by the fly in such behavioural closed-loop experiments were repeatedly replayed to an identified neuron in the fly's pathway for processing visual motion information. In this way it could be shown, that the SIRC can be decoded most reliably in the individual real-time responses of the neuron, if the activity is smoothed on a relatively coarse time scale, i.e. within time windows of $40-100 \mathrm{~ms}$. At a finer temporal resolution the reliability of neuronal information processing decreases due to stochastic fluctuations in the spike activity. Such large time windows for decoding the SIRC appear to be plausible also from another perspective. For instance, an $80 \mathrm{~ms}$ integration window would allow to recover frequencies in the SIRC of up to $6.25 \mathrm{~Hz}$. Higher frequency components. however, do not contribute much to the SIRC (see Fig. 2B). Thus high-frequency variations in spike activity can be safely smoothed out without much deteriorating the representation of the behaviourally generated stimuli.

The conclusion that the stimulus-induced component contained in the responses of the analysed neuron in the fly's visual motion pathway can be decoded best by temporal integration over some 40 $100 \mathrm{~ms}$ requires some further comments. (i) This conclusion is not meant to imply that the fly's motion-sensitive neurons cannot respond to rapid changes in pattern velocity. Rather, it has been shown in various studies that they respond to some extent to oscillating stimulus patterns with frequencies above $10 \mathrm{~Hz}$ (Egelhaaf and Reichardt, 1987; Haag and Borst, 1996). However, such high-frequency stimuli do not play a major role in optomotor course stabilization. (ii) The perform- ance of the fly motion-sensitive neuron in representing information about the SIRC does not only depend on the size of the time window but also on its shape. Of course, the type of window used in the present study that weighs all events equally strongly does not appear very plausible from a physiological point of view. Moreover, it is likely that there are differently shaped time windows that lead to a more reliable representation of the SIRC by the individual responses than those used in the present analysis. However, there is no reason to assume that the shape of the time window chosen in the present study would affect our general conclusion, that in the fly motionsensitive neuron behaviourally relevant motion stimuli, as obtained in the context of optomotor course stabilization, can be resolved best at a time scale where the timing of individual spikes does not matter. (iii) The optimal size of the time window depends on the dynamic properties of the stimuli. In fact, temporal integration on a smaller time scale than inferred in the present study was found to be optimal for decoding a white noise signal from the real-time responses of the H1-neuron (Bialek et al., 1991). This is not surprising since the noise signal was band-limited at $1 \mathrm{kHz}$ and thus contained information at much higher temporal frequencies than occur in stimuli that are generated by the fly in a behavioural closed-loop situation. In this context, it should be emphasized again that the motion traces generated in the context of optomotor course stabilization and used here as stimuli, did not contain much power above $6-10 \mathrm{~Hz}$ (see Fig. 2). The temporal characteristics of the retinal image displacements may differ considerably in behavioural contexts other than optomotor course stabilization such as, e.g. obstacle avoidance or pursuit of potential mates. On what time scale the relevant information has to be decoded in these behavioural situations needs to be elucidated in future experiments. (iv) Our conclusion is based on an information theoretic analysis. It is therefore not meant to imply that the fly actually employs such a decoding strategy. We are currently addressing at least part of this important problem by characterizing how the information conveyed by a motion-sensitive neuron is decoded by its postsynaptic cells. In this context it needs to be emphasized that it is not necessarily the task of the postsynaptic neurons to represent the SIRC as well as possible and thus to act simply as a channel transmitting information. Rather the task of neurons and neuronal circuits usually is to process the incoming information in order to explicitly extract certain aspects of a stimulus that are only implicitly contained in the incoming signals (e.g. extract motion information from the spatio-temporal changes in luminance perceived by the photoreceptors). Depending on the task such processing might require to treat the presynaptic signals on a time scale different from the one which is optimal to represent the SIRC.

\section{How does the present approach relate to other approaches?}

In order to assess the reliability of neuronal information processing, we recorded sequences of spike trains induced by identical stimuli. On this basis, we could determine those components of the individual responses that are induced by the stimulus and those which have a stochastic origin. The relative size of these two response components allowed us to infer how much information about the time-course of the stimulus-induced response component is transmitted by individual spike trains and, thus, how reliably information is processed up to the level of an identified motion-sensitive neuron of the fly that plays a role in tuning other neurons to particular types of optic flow. In contrast to related studies (e.g. Eckhorn and Pöpel, 1974; Theunissen and Miller, 1991), it was not intended by the present account to find out the relationship between individual responses and the stimuli by which they were elicited.

Moreover, our approach to assessing the reliability of neural 
information processing is in clear distinction to approaches that are aimed at reconstructing the stimulus from an individual time-dependent spike train and, in this way, to find out what the neuron codes for (for review, see Bialek 1990). This so-called reverse reconstruction method has been applied to a variety of sensory systems (Rieke $e t a l$, 1991; Roddey and Jacobs, 1996; Theunissen et al., 1996), including the H1-neuron of the fly (Bialek et al., 1991). With respect to the $\mathrm{H}$ 1-neuron, a linear filter was derived and used to estimate from individual spike trains the velocity of the motion stimulus by which they were elicited. Deviations of the estimated stimulus from the original velocity traces were interpreted as to reflect the effective noise level and fault tolerance of neural computation' (p. 1854 in Bialek et al., 1991). With respect to the latter conclusion, some qualifications need to be made. It has long been known that the motion-sensitive neurons of the fly do not encode stimulus velocity as the only stimulus parameter. Rather, their responses depend on other aspects of the stimulus as well, such as its spatial frequency content and contrast (for review see Borst and Egelhaaf, 1989; Egelhaaf and Borst, 1993b). Moreover, even for a given stimulus pattern, the time-course of the SIRC is not linearly related to pattern velocity but rather depends in a non-linear way on the weighted sum of the instantaneous velocity and all its higher-order temporal derivatives. The responses are approximately linearly related to pattern velocity only for small displacements and slow velocity changes (Egelhaaf and Reichardt, 1987). Hence, deviations of the estimated stimulus, as obtained by the reverse reconstruction method from pattern velocity, can have two sources. (i) Stochastic response components may lead to an imperfect reconstruction. (ii) The relationship between stimulus velocity and SIRC may be not sufficiently linear. Since these two factors cannot be disentangled by means of the reconstruction procedure, the latter can hardly provide useful information about how the reliability of neuronal coding is deteriorated by noise.

\section{Temporal encoding in the nervous system}

Although it is generally agreed that neurons signal information through changes in their electrical activity, the 'neural code' by which information is represented in this neural activity still remains elusive. Thus, it is not surprising that the search for the 'neural code', which is used by an organism in a particular behavioural or perceptual task, is getting increasingly fashionable (e.g. Shadlen and Newsome, 1994; Theunissen and Miller, 1995; König et al., 1996). An important aspect of the current still highly controversial debate is the question on what time scale temporal spike patterns can convey significant information (Shadlen and Newsome, 1994, 1995; Softky, 1995; König et al., 1996). Quite generally, this scale depends essentially on how precisely spikes are timed contingent upon a given pattern of synaptic input and thus on the resulting spatio-temporal pattern of postsynaptic potentials. How reliably neuronal information can be encoded is mainly a question of how deterministic neuronal processes are and to what extent they are affected by stochastic processes that lead to a jitter in the timing of spikes contingent upon a given input of the cell.

The size of the stochastic component has been estimated for a wide range of neurons of various species. Although the actual noise strongly depends on the time window in which the spikes are counted and thus the estimates vary between the studies, it is clear that the variance of the spike count may be in the range of the mean spike count or even larger (Tolhurst et al., 1983; Vogels et al., 1989; Miller et al., 1991; Britten et al., 1993). The H1-cell in the fly's visual motion pathway lies at the lower end of this range with respect to its variability (Warzecha, 1994). Although the H1-cell is thus not a particularly noisy neuron, its spikes are not precisely time-locked, at least on a millisecond time scale, to motion stimuli as are generated by the fly itself under behavioural closed-loop conditions. Nevertheless, the discharge rate of motion-sensitive neurons in the fly modulates in a time-locked fashion to the time-course of external visual inputs at a time scale of tens to hundreds of milliseconds (see Fig. 1) in a similar way as described, for instance, for interneurons in the monkey cortical area MT (Shadlen and Newsome, 1994); however, these neurons may respond to favourable transient stimuli somewhat more precisely, i.e. with a jitter of $<10 \mathrm{~ms}$ (Bair and Koch, 1995).

Despite the considerable variability in neuronal activity as described for many neurons, several studies using a range of different approaches suggest the importance of precise, complex single-spike computations on a time scale well below the average interspike interval. In particular, it has been proposed that a neuron could signal reliably the occurrence of certain combinations of presynaptic events with a temporal fidelity in the order of a millisecond or less (Softky, 1995; König et al., 1996). Moreover, as long as the input signal is sufficiently transient, cortical neurons in a slice preparation may produce spike trains with a timing reproducible to $<1 \mathrm{~ms}$, as could be shown by injecting a white noise current stimulus (Mainen et al., 1995). In addition, spike patterns with a temporal precision in the range of only a few milliseconds may occur much more frequently than can be expected if spike timing were random (Strehler and Lestienne, 1986; Abeles et al., 1993). And of course, some sensory systems such as the auditory system of the barn owl or the electrosensory system of electric fish rely on precise neural responses well below the millisecond range to localize an object or communicate with conspecifics (for review see Konishi, 1991; Carr, 1993). Finally, spiking in a pair of identified motion-sensitive neurons in the visual system of the fly which receive their input from the same set of retinotopic input elements was found to be mutually time-locked on a millisecond time-scale. Since this high degree of synchronicity is not due to stimulus-induced modulations of the synaptic input to these neurons but rather due to some unknown source, it does not conflict with the variable responses of these neurons to repetitive presentation of identical stimuli (Warzecha et al., 1997; A.-K. Warzecha, in preparation). It should be noted that, apart from the electric fish and the barn owl, the functional significance of precisely timed spikes is not yet experimentally established in any other of the above mentioned systems. Hence, it cannot be decided here whether the described neuronal precision has any relevance for neuronal information processing or whether it is just an epiphenomenon of some biophysical properties of nerve cells and their interactions.

The few examples for which it has been possible to relate the precision of neuronal events to the behavioural performance of the whole animal may suggest that the precision of neural coding is adapted by evolution to the requirements of the respective system. For instance, for a barn owl as a nocturnal predator that relies on acoustic cues to detect its prey there is no other possibility than to exploit temporal cues with a precision in the range of a few microseconds. On the other hand, for an animal such as the fly it might be advantageous in visual orientation behaviour to temporally integrate the incoming signals as long as is allowed by the temporal dynamics of the stimuli as are generated by the fly's own actions and reactions. Interestingly, integration times of a similar size as found here for a fly motion-sensitive neuron were also concluded to be most appropriate to decode the activity of neurons in the visual as well as in the inferotemporal cortex of the monkey (Heller et al., 1995; Victor and Purpura 1996). Also, in these systems the precise timing of individual spikes does not appear to matter for the processing of information. A similar conclusion has been drawn in combined behavioural and electrophysiological experiments on the escape 
system of the cockroach, which mediates turning reactions away from a potential source of danger. It was found that the cockroach integrates over a couple of spikes before eliciting an escape response rather than relying on the tine temporal patterning of a spike sequence (Liebenthal et al., 1994).

In conclusion, on what time scale relevant information is conveyed can only be assessed if the computational needs of the respective system are known. If there is any chance to find out what aspects of neural activity encode significant information and on what time scale this is accomplished, the analysis at the neuronal level has to be guided and constrained by the performance of the animal as it manifests itself at the behavioural level.

\section{Acknowledgements}

We are grateful to J. Eikermann for her help in performing the experiments. V. Dürr, R. Kern and B. Kimmerle as well as anonymous referees made many useful suggestions on drafts of the manuscript and thus helped to improve the paper. During part of this study A.-K. W. was supported by a grant of the Max-Planck Society. We are indebted to $\mathrm{K}$. Götz who made this support possible.

\section{Abbreviations}

SIRC stimulus-induced response component

\section{References}

Abeles, M., Bergman, H.., Margalit, E. and Vaadia, E. (1993) Spatiotemporal firing patterns in the hrontal cortex of behaving monkeys. J. Neurophysiol, 70, 1629-1638.

Allen, C. and Stevens, C. F. (1994) An evaluation of causes for unreliability of synaptic transmission. Proc. Natl Acad. Sci. USA, 91, 10380-10383.

Bair, W. and Koch, C. (1996) Temporal precision of spike trains in extrastriate cortex of the behaving macaque monkey. Neural Computation, 8, 11851201.

Bialek, W. (1990) Theoretical physics meets experimental neurobiology. In Jen, E. (ed.), 1989 Leitures in Complex Systems, SFI Studies in the Sciences of Complexity, vol. 2. Addison-Wesley, pp. 513-595.

Bialek, W., Rieke, F., Ruyter van Steveninck, R. D. and Warland, D. (1991) Reading a neural code. Science, 252, 1854-1857.

Borst, A. and Egelhaal, M. (1989) Principles of visual motion detection. Trends Neurosci., 12. 297-306.

Bouman, M. A., van de Grind, W. A. and Zuidema, P. (1985) Quantum fluctuations in vision. In Wolf, E. (ed.), Progress in Optics, Vol. XXII, North Holland, Amsterdam. New York, Oxford, Tokyo, pp. 79-144.

Britten, K. H., Shadlen. M. N., Newsome, W. T. and Movshon, J. A. (1993) Responses of neurons in macaque MT to stochastic motion signals. Vis. Neurosci, 10, 1157-1169.

Carr, C. E. (1993) Processing of temporal information in the brain. Annu. Rev. Neurosci, 16, 223-243.

Eckhorn, R. and Pöpcl, B. (1974) Rigorous and extended application of information theory to the afferent visual system of the cat. I. Basic concepts. Kybernetik, 16, 191-200.

Egelhaaf, M. and Borst, A. (1993a) A look into the cockpit of the fly: visual orientation, algorithms, and identified neurons. J. Neurosci., 13, 4563-4574.

Egelhaaf, M. and Borst, A. (1993b) Movement detection in arthropods. In Wallman, J. and Miles, F. A. (eds.), Visual Motion and its Role in the Stabilization of Gaze. Elsevier, Amsterdam.

Egelhaaf, M. and Reichardt, W. (1987) Dynamic response properties of movement detectors: Theoretical analysis and electrophysiological investigation in the visual system of the fly, Biol. Cybern., 56, 69-87.

Egelhaaf, M., Hausen. K., Reichardt, W. and Wehrhahn, C. (1988) Visual course control in thies relies on neuronal computation of object and background motion. Trends Neurosci., 11, 351-358.

Gestri, G., Mastebroek, H. A. K. and Zaagman, W. H. (1980) Stochastic constancy, variability and adaptation of spike generation: performance of a giant neuron in the visual system of the fly. Biol. Cybern., 38, 31-40.

Haag, J. and Borst, A. (1996) Amplification of high frequency synaptic inputs by active dendritic nembrane processes. Nature, 379, 639-641.
Hausen, K. (1981) Monocular and binocular computation of motion in the lobula plate of the fly. Verh. Dtsch. Zool. Ges., 74, 49-70.

Hausen, K. (1982) Motion sensitive interneurons in the optomotor system of the fly. I. The horizontal cells: structure and signals. Biol. Cybern., 45, 143-156.

Hausen, K. and Egelhaaf, M. (1989) Neural mechanisms of visual course control in insects. In Stavenga, D. and Hardie, R. (eds.), Facets of Vision. Springer-Verlag, Berlin, pp. 391-424.

Heller, J., Hertz, J. A., Kjaer, T. W. and Richmond B. J. (1995) Information flow and temporal coding in primate pattern vision. J. Comput. Neurosci., 2, 175-193.

Heisenberg. M. and Wolf, R. (1984) Vision in Drosophila. Springer-Verlag, Berlin.

Hille, B. (1992) Ionic Channels of Excitable Membranes, 2nd edn. Sinauer Associales, Sunderland.

Kimmerle, B., Srinivasan, M. V. and Egelhaaf, M. (1996) Object detection by relative motion in freely flying flies. Naturwissenschaften, 83, 380-381

Konishi, M. (1991) Deciphering the brain's codes. Neur. Comput., 3, 1-18.

König, P., Engel, A. K. and Singer, W. (1996) Integrator or coincidence detector? The role of the cortical neuron revisited. Trends Neurosci, 19, 130-137.

Land, M. F. and Collett, T. S. (1974) Chasing behaviour of houseflies (Fannia canicularis). A description and analysis. J. Comp. Physiol., 89, 331-357.

Liebenthal, E., Uhlmann, O. and Camhi, J. M. (1994) Critical parameters of the spike trains in a cell assembly: coding of turn direction by giant interneurons of the cockroach. J. Comp. Physiol. A, 174, 281-296.

Mainen, Z. F., Joerges, J., Huguenard, J. R. and Sejnowski, T. J. (1995) A model of spike initiation in neocortical pyramidal neurons. Neuron, 15 , 1427-1439.

Miller, J. P., Jacobs, G. A. and Theunissen, F. E. (1991) Representation of sensory information in the cricket cercal sensory system. I. Response properties of the primary interneurons. J. Neurophysiol., 66, 1680-1689.

Newsome, W. T., Britten, K. H., Movshon, J. A. and Shadlen, M. (1989) Single neurons and the perception of visual motion. In Lam, D. M.-K. and Gilbert, C. D. (eds), Neural Mechanisms of Visual Perception. Gulf, Houston. pp. 171-198

Reichardt, W. and Poggio, T. (1976) Visual control of orientation behaviour in the fly. Part I. A quantitative analysis. Q. Rev. Biophys., 9, 311-375.

Rieke, F., Owen, W. G. and Bialek, W. (1991) Optimal filtering in the salamander retina. In Lippman, R. P., Moody, D. S. and Touretzky, D. S. (eds), Advances in Neural Information Processing 3. Morgan Kaufmann, San Mateo, CA, pp. 377-383.

Roddey, J. C. and Jacobs, G. A. (1996) Information theoretic analysis of dynamical encoding by filiform mechanoreceptors in the cricket cercal system. J. Neurophysiol., 75, 1365-1376.

Ruyter van Steveninck. R. D. and Bialek, W. (1988) Real-time performance of a movement-sensitive neuron in the blowfly visual system: coding and information transfer in short spike sequences. Proc. R. Soc. Lond. B., 234, 379-414.

Ruyter van Steveninck, R. D. and Bialek, W. (1995) Reliability and statistical efficiency of a blowfly movement-sensitive neuron. Philos. Trans. $R$. Soc. Lond. B, 348, 321-340.

Shadlen, M. N. and Newsome, W. T. (1994) Noise, neural codes and cortical organization. Curr. Opin. Neurobiol., 4, 569-579.

Shadlen, M. N. and Newsome, W. T. (1995) Is there a signal in the noise? Curr. Opin. Neurobiol., 5, 248-250.

Shadlen, M. N., Britten, K. H., Newsome, W. T, and Movshon, J. A. (1996) A computational analysis of the relationship between neuronal and behavioral responses to the visual motion. J. Neurosci., 16, 1486-1510.

Shannon, C. E. and Weaver, W. (1949) The Mathematical Theory of Communication. The University of Illinois Press, Urbana.

Softky, W. R. (1995) Simple codes versus efficient codes. Curr. Opin. Neurobiol., 5, 239-247.

Strehler, B. L. and Lestienne, R. (1986) Evidence on precise time-coded symbols and memory of patterns in monkey cortical neuronal spike trains. Proc. Natl Acad. Sci. USA, 83, 9812-9816.

Theunissen, F. E. and Miller, J. P. (1991) Representation of sensory information in the cricket cercal sensory system. II. Information theoretic calculation of system accuracy and optimal tuning-curve widths of four primary interneurons. J. Neurophysiol., 66, 1690-1703.

Theunisscn, F. and Miller, J. P. (1995) Temporal encoding in nervous systems: a rigorous definition. J. Comp. Neurosci., 2, 149-162.

Theunissen, F., Roddey, J. C., Stufflebeam, S., Clague, H. and Miller, J. P. (1996) Information theoretic analysis of dynamical encoding by four identified primary sensory interneurons in the cricket cercal system. $J$. Neurophysiol., 75, 1345-1364. 
1374 Reliability of a fly motion-sensitive neuron

Tolhurst, D. J., Movshon, J. A. and Dean, A. F. (1983) The statistical reliability of signals in single neurons in cat and monkey visual cortex. Vision Res., 23, 775-785.

Victor, J. D. and Purpura, K. P. (1995) Nature and precision of temporal coding in visual cortex: a metric-space analysis. J. Neurophysiol, 76, 1310-1326.

Vogels, R., Spilcers, W. and Orban, G. A. (1989) The response variability of striate cortical neurons in the behaving monkey. Exp. Brain Res., 77, $432-436$
Wagner, H. (1986) Flight performance and visual control of flight of the free-flying housefly (Musca domestica). III. Interactions between angular movement induced by wide- and smallfield stimuli. Philos. Trans. R. Soc Lond. B, 312, 581-595.

Warzecha, A.-K. (1994) Reliability of Neuronal Information Processing in the Motion Pathway of the Blowflies Calliphora erythrocephala and Lucilia cuprina. Doctoral Disseration, Universität Tübingen.

Warzecha, A.-K. and Egelhaaf, M. (1996) Intrinsic properties of biological movement detectors prevent optomotor control system from getting unstable. Philos. Trans. R. Soc. Lond. B, 351, 1579-1591. 